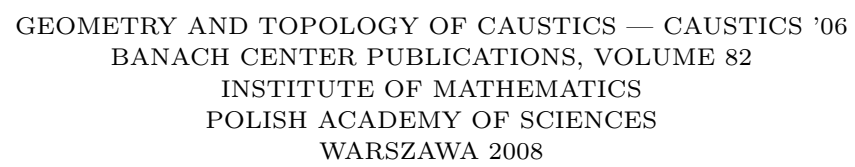

\title{
QUASI SINGULARITIES
}

\author{
VLADIMIR M. ZAKALYUKIN \\ Department of Mathematical Sciences, The University of Liverpool \\ Liverpool L69 7ZL, UK \\ and \\ Faculty of Mechanics and Mathematics, Moscow State University \\ 119992, Moscow, Russia \\ E-mail: zakalyu@mail.ru
}

\begin{abstract}
A classification of simple equivalence classes of function germs with respect to new relations is given. The equivalence relation is similar but weaker than the right action of diffeomorphisms which preserve the boundary. It is used in classifying Lagrange projections with boundary. The simple classes of function germs with respect to the equivalence similar to fibration preserving action are also discussed.
\end{abstract}

1. Introduction. Thom-Boardman classes determine finite stratification of arbitrary order jet spaces of mappings, while the orbits of standard group actions form simple discrete stratification only for jets of low order. To get examples of stratifications with extra simple strata we introduce some new equivalence relations determined by conditions imposed on jets of functions. They are more rough than standard equivalences, and keep trace only of some invariants of standard singularities. However they have useful applications.

We describe quasi-boundary singularities of functions and quasi-projections. Quasiboundary equivalence plays an intermediate role between the standard right equivalence of functions (diffeomorphisms action on the source space) and the boundary equivalence (right action of diffeomorphisms, which preserve a given boundary hypersurface).

Singularities of quasi-projections are equivalence classes which are finer than right equivalence orbits but are larger than the orbits under the group of the diffeomorphisms which preserve a given fibration with line fibers.

In each case we classify discrete (simple) equivalence classes and describe corresponding discriminant and bifurcation diagrams.

2000 Mathematics Subject Classification: 58C27, 58K40.

Key words and phrases: classification of function germs, boundary singularities.

The paper is in final form and no version of it will be published elsewhere. 
In spite of rather artificial nature of the definitions, quasi-boundary singularities have natural applications.

Their discriminants describe bifurcation of critical point of a function (for example, its global extremum) inside and on certain domain with a boundary.

Another even more valuable application of quasi-boundary singularities arises in symplectic geometry. We consider Lagrangian mappings of a Lagrangian manifold with a boundary. A boundary is an isotropic hypersurface in the Lagrangian manifold. These pairs are natural in various singularity theory applications to differential equations and variational problems. Isotropic submanifold plays the role of the initial data set and Lagrangian submanifold is the solution of Hamilton-Jacobi equation with the initial data. Similar problems were studied in $[5,6]$.

The pair is embedded into the phase space which usually is the cotangent bundle of the configuration space. Simple classes of the projections to the configuration space of these pairs are exactly simple quasi-boundary classes.

Arnold's boundary singularities are related to projections of the pairs of Lagrangian submanifolds ( of dimension $n$ ) which have $n$-1-dimensional regular intersection and are transversal in complementary directions [1]. Quasi-equivalence relation (introduced for generating families) keeps information only on one Lagrangian submanifold and on its intersection with the second component.

The list of simple classes contains new classes and series of classes corresponding to non-simple ordinary boundary singularities. Similar properties are valid for quasiprojections.

We hope that exploring other non-standard equivalences will help to understand better the geometry beyond standard simple classes in various singularity theory problems.

2. Pseudo- and quasi-boundary singularities. Ordinary boundary singularities, introduced by $\mathrm{V}$. Arnold, are the orbits in the space of smooth functions $f: \mathbf{R}^{n} \rightarrow \mathbf{R}$ of the action of the diffeomorphisms of $\mathbf{R}^{n}$ which preserve a distinguished hyperplane $B$ (called boundary). Here we consider germs of smooth functions $f:\left(\mathbf{R}^{n}, 0\right) \rightarrow \mathbf{R}$ on $\mathbf{R}^{n}=\{(x, y)\}, x \in \mathbf{R}^{n-1}, y \in \mathbf{R}$ with the boundary $B=\{y=0\}$.

Definition 2.1. Two functions $f_{1}, f_{2}$ are called pseudo-boundary right equivalent if there is a diffeomorphism $\theta: \mathbf{R}^{n} \rightarrow \mathbf{R}^{n}$ such that $f_{1}=f_{2} \circ \theta$ and if $b \in B$ is a critical point of $f_{1}$ then $\theta(b) \in B$, also if $c \in B$ is a critical point of $f_{2}$ then $\theta^{-1}(c) \in B$.

Obviously, this is an equivalence relation. We will denote by $P_{f}$ the equivalence class of a germ $f$ and call it the pseudo-orbit of $f$. As usual in singularity theory we are interested in connected components of orbits. This leads to an infinitesimal characterization of the equivalence. Differentiating upon parameter all deformations within the pseudo-orbit of a given germ $f$ we obtain the tangent space $T P_{f}$ at $f$ to the orbit $P_{f}$. Consider germs of function in $x, y$ at the origin, and denote by $\mathbf{C}_{x, y}$ the algebra of these germs.

Clearly, the tangent space is given by the formula

$$
T\left(P_{f}\right)=\frac{\partial f}{\partial x} \mathbf{C}_{x, y}+\frac{\partial f}{\partial y}\left\{y \mathbf{C}_{x, y}+\operatorname{Rad}\left(J_{f}\right) \mathbf{C}_{x, y}\right\},
$$

where $\operatorname{Rad}\left(J_{f}\right)$ is the radical of the Jacobi ideal of $f$. 
REMARK. Unfortunately, this relation does not satisfy the properties of a geometrical subgroup of equivalences in the J. Damon sense [2]. In particular, the versality theorem can fail. To avoid this difficulty we use a subspace of the tangent space, which behaves regularly when the function depends on extra parameters. Namely take the following sub-module

$$
T Q_{f}=\frac{\partial f}{\partial x} \mathbf{C}_{x, y}+\frac{\partial f}{\partial y}\left\{y \mathbf{C}_{x, y}+J_{f} \mathbf{C}_{x, y}\right\} \subset T P_{f},
$$

as the set of admissible infinitesimal deformations of a function, and introduce the respective notion $Q_{f}$ of the class of a equivalence relation which is finer than the pseudoequivalence.

Definition 2.2. A vector field on $x, y$ space is called $f$-admissible if its $\frac{\partial}{\partial y}$ component belongs to the ideal $y \mathbf{C}_{x, y}+J_{f} \mathbf{C}_{x, y}$ in the algebra $\mathbf{C}_{x, y}$ of smooth functions in $x, y$.

Two functions $f_{1}, f_{2}$ are called quasi-boundary equivalent if there is a family of diffeomorphisms $\theta_{t}: \mathbf{R}^{n} \rightarrow \mathbf{R}^{n}$ continuously and piecewise smoothly depending on parameter $t \in[1,2]$ such that $\theta_{2}$ is the identity mapping, $f_{1}=f_{2} \circ \theta_{1}$, and for any $t \in[1,2]$ the vector field $V=\frac{d \theta_{t}}{d t} \circ \theta_{t}^{-1}$ generated by $\theta_{t}$ is $f_{t}$-admissible for the function $f_{t}=f_{2} \circ \theta_{t}$. The family of functions $f_{t}$ being the homotopy between $f_{1}$ and $f_{2}$ is called admissible.

Apparently, quasi-boundary equivalence implies pseudo-boundary equivalence.

Define pseudo-boundary and quasi-boundary right-plus equivalence modifying the definition 2.1 and admitting extra addition of functions with constants.

Similarly, introduce the contact version of these definitions. Two hypersurfaces $f_{1}=0$, and $f_{2}=0$ are called pseudo-boundary equivalent if they are diffeomorphic via a diffeomorphism which maps critical points of the first hypersurface which are in the boundary to those of the second hypersurface and vice versa. The respective definition of quasiboundary equivalence of hypersurfaces is straightforward. For shortness, the right quasiboundary equivalence will be called below just quasi-equivalence.

The versality theorem for quasi-equivalence holds. As usual, the miniversal deformation of a function $f(x, y)$ germ is a sum of an organizing center $f(x, y)$ with the linear combination of the functions whose classes form a basis over $\mathbf{R}$ of the quotient space $\mathbf{C}_{x, y} / T Q_{f}$.

To determine simple classes with respect to quasi-equivalence we start with the following auxiliary remarks.

The quasi-classification of critical points outside the boundary coincides with the ordinary $R$-equivalence. In particular, classes $A_{k}, D_{k}, E_{k}$ form the list of simple quasiclasses outside the boundary. So we consider only the classes of critical points in the boundary (at the origin).

Standard Arnold's boundary equivalence (right action of diffeomorphisms preserving the boundary) imply quasi-equivalence. So simple Arnold's boundary classes $B_{k}, C_{k}, F_{4}$ remain simple for quasi-classification, but some classes can merge together.

A priori it is not clear why even the jets of quasi-orbits are submanifolds of jetspaces. Tangent spaces of admissible deformations can form a non-integrable distribution. Fortunately, the jets of orbits of simple classes listed below are submanifolds as can be 
seen from the proof of the theorem. The commutator of two $f$-admissible vector fields is not $f$-admissible in general. To tackle quasi-equivalence special techniques are required.

Assuming the critical distinguished point remains at the origin for any parameter value of the deformation we can consider only admissible vector fields which vanish at the origin. In a range of cases this permits to show that jets of some order of quasi-orbits coincide with jets of standard Arnold's boundary orbits.

In many cases we can find an appropriate prenormal form of a germ.

LEMma 2.3. If $\Phi_{t}(x, y)$ is an admissible deformation, and $(x, y) \mapsto\left(X_{t}(x, y), Y_{t}(y)\right)$ is a family of diffeomorphisms of $\mathbf{R}^{n}$ which preserve the fibration $(x, y), 0 \mapsto y, 0$ then $G_{t}(x, y)=\Phi_{t}\left(X_{t}(x, y), Y_{t}(y)\right)$ is also an admissible family.

Proof. The claim that the deformation $\Phi_{t}(X, Y)$ is admissible means that

$$
\begin{aligned}
-\frac{\partial \Phi_{t}(X, Y)}{\partial t} & \\
= & \frac{\partial \Phi_{t}(X, Y)}{\partial X} \dot{X}+\frac{\partial \Phi_{t}(X, Y)}{\partial Y}\left(Y A(X, Y, t)+B(X, Y, t) \frac{\partial \Phi_{t}(X, Y)}{\partial Y}\right)
\end{aligned}
$$

with some smooth functions $A, B, \dot{X}_{i}$.

The matrix $\frac{\partial X}{\partial x}$ is invertible, $\frac{\partial Y}{\partial y} \neq 0$, and $\frac{\partial \Phi_{t}}{\partial x}=\frac{\partial \Phi_{t}}{\partial X} \frac{\partial X}{\partial x}, \frac{\partial \Phi_{t}}{\partial y}=\frac{\partial \Phi_{t}}{\partial X} \frac{\partial X}{\partial y}+\frac{\partial \Phi_{t}}{\partial Y} \frac{\partial Y}{\partial y}$. The function $Y$ is divisible by $y$. Hence the decomposition can be written in the form

$$
\begin{aligned}
& -\frac{\partial \Phi_{t}(X(x, y), Y(y))}{\partial t}=\frac{\partial \Phi_{t}(X(x, y), Y(y))}{\partial x} \widetilde{X} \\
& \quad+\frac{\partial \Phi_{t}(X(x, y), Y(y))}{\partial y}\left(Y \widetilde{A}(x, y, t)+\widetilde{B}(x, y, t) \frac{\partial \Phi_{t}(X(x, y), Y(y))}{\partial y}\right)
\end{aligned}
$$

with some smooth functions $\widetilde{X}, \widetilde{A}, \widetilde{B}$ holds. This means that the family $G_{t}$ is admissible.

LEMMA 2.4. If $G_{t}(x, y)$ is an admissible family then for an arbitrary smooth function $H(t, x, y)$ the family $\widetilde{G}_{t}(x, y)=G_{t}(x, y)+H\left(\frac{\partial G_{t}}{\partial y}\right)^{2}$ is also admissible and $\widetilde{G}_{t}$ is quasiequivalent to $G_{t}$ for each value of $t$.

The proof is a straightforward verification of persistence of the homologic decomposition based on the fact that $\frac{\partial \widetilde{G}_{t}}{\partial y}=\frac{\partial G_{t}}{\partial y} K(x, y, t)$, and $K \neq 0$.

Lemma 2.5 (Stabilization). If the rank of the second differential $d_{0}^{2}\left(\left.f\right|_{y=0}\right)=k$ then the function germ $f(x, y)$ is quasi-equivalent to $\sum_{i=1}^{k} \pm x_{i}^{2}+\widetilde{f}(\widetilde{x}, y), \widetilde{x} \in \mathbf{R}^{n-1-k},\left.\widetilde{f}\right|_{y=0} \in M_{\widetilde{x}}^{3}$. For quasi-equivalent $f$ germs, the respective reduced $\widetilde{f}$ germs are also quasiequivalent. The reduction of an admissible family remains admissible.

In fact, the required reduction can be performed using the ordinary group of diffeomorphisms preserving the boundary. So we apply Lemma 2.3.

THEOREM 2.6. A simple quasi-boundary singularity class is either one of the standard $A_{k}, D_{k}, E_{k}$ classes outside the boundary or a class of a stabilizations of one of the follow- 
ing germs

$$
\begin{aligned}
& B_{k}: \pm x^{2}+ \pm y^{k}, \quad k \geq 2 \quad k \\
& F_{k, l}: \pm\left(y+x^{k}\right)^{2} \pm x^{l+1}, \quad 2 \leq k \leq l \quad k+l \\
& D_{k, l}: \pm\left(y+x_{1}^{k}\right)^{2}+x_{1} x_{2}^{2} \pm x_{1}^{l-1}, \quad 2 \leq k \leq l-1, l \geq 4 \quad k+l+1
\end{aligned}
$$

The orbit codimensions in the space of germs are shown in the right column.

REMARKS. 1. The class $B_{2}: x^{2}-y^{2}$ has another equivalent form $C_{2}: x y$ (which represents a single quasi-class containing all ordinary $C_{k}$ boundary classes in the plane).

2. The classes $F_{k, k}$ have equivalent forms $\pm y^{2} \pm x^{k+1}$. In particular, $F_{2,2}: y^{2}+x^{3}$ coincides with $F_{4}$ ordinary boundary singularity class.

3. The classes $D_{l-1, l}$ have equivalent forms $\pm y^{2}+x_{1} x_{2}^{2} \pm x_{1}^{l-1}$.

4. The fencing (non-simple) classes are either of infinite codimension (adjacent to all $B_{k}$-classes $)$ or the following uni-modal $(a \in \mathbf{R})$ classes

$$
\widetilde{S}_{5}: y^{3}+x^{3}+a y^{2} x \quad \text { and } \quad \widetilde{E}_{2,6}: \pm\left(y+x_{1}^{2}+a x_{1} x_{2}\right)^{2}+x_{1}^{4}+x_{2}^{3} y
$$

of codimensions respectively 5 and 9 .

5. The adjacency of lower codimension classes is shown in the table

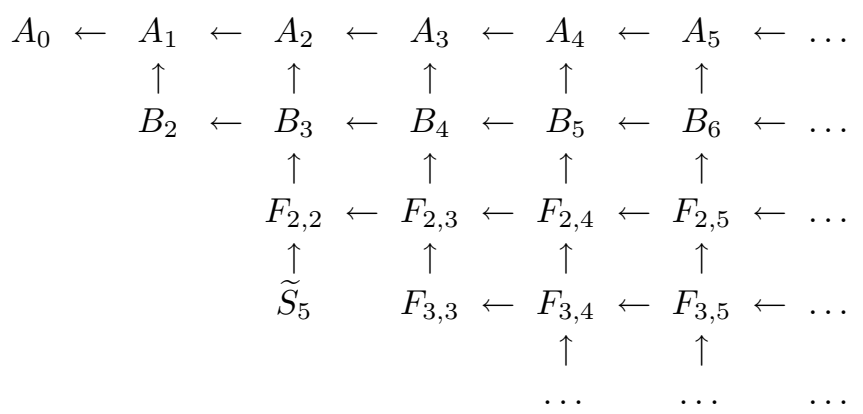

Any adjacency between simple classes is a consequence of $B_{k} \leftarrow F_{2, k-1}, F_{l, m} \leftarrow F_{l, m+1}$, $F_{l, m} \leftarrow F_{l+1, m}$, and also $F_{k+1, l-1} \leftarrow D_{k, l}, D_{l, m} \leftarrow D_{l, m+1}, D_{l, m} \leftarrow D_{l+1, m}$. The class $E_{2,6}$ is adjacent to $D_{2,5}$.

6. If $n \geq 3$ the $F_{k, l}$ germ have a stabilization which is quasi-equivalent to

$$
\widetilde{F}_{k, l}: y x_{1}+x_{1} x_{2}^{k}+x_{2}^{l+1}
$$

(provided that $2 \leq k \leq l$ ).

7. For $n \geq 4$ simple quasi-orbit $D_{k, l}$ has a stabilization which is quasi-equivalent to

$$
\widetilde{D}_{l, m}: y x_{1}+x_{1} x_{2}^{l}+x_{2} x_{3}^{2}+x_{2}^{m-1}
$$

(for $2 \leq l \leq m-2,4 \leq m$ ).

8. Classification with respect to contact quasi-equivalence has the same list of simple classes as $\mathbf{R}$ quasi-equivalence due to weighted homogeneity.

9. Also the $\mathbf{R}+$ quasi-equivalence simple list coincides with the list of the theorem. Deleting the constant terms from the quasi-equivalence versal deformation we get $\mathbf{R}+-$ versal deformation. 
10. Notice that classes $F_{3,3}$ and $F_{2,3}$ are pseudo-equivalent but not quasi-equivalent. In fact, the transformation $x, y \mapsto x, y+x^{2}$ is pseudo-equivalence between $y^{2}+x^{4}$ of $F_{3,3}$ type and $\left(y+x^{2}\right)^{2}+x^{4}$ which is of $F_{2,3}$. However their codimensions of quasi-tangent spaces are different, and the classes are not quasi-equivalent.

The bifurcation diagram (with respect to quasi-equivalence) of a function germ deformation consists of two components. The first is the ordinary discriminant of the deformation $f$ given by the equations $d f=0, f=0$. The second is its subset satisfying the extra equation $y=0$. For simple singularities the first is the cylinder over the standard discriminant of a simple hypersurface singularity which is isomorphic to the discriminant of the corresponding group generated by reflections. This is a hypersurface in parameter space. The second component has codimension 2 in the ambient space.

The caustic of the $\mathbf{R}+$-versal deformation consists of two hypersurface strata. The first one is the ordinary caustics of $f$ - that is the subset of parameters of the $\mathbf{R}+$-versal deformation, which corresponds to the functions with non-Morse critical points. The other one is the projection to the base of $\mathbf{R}$ +-deformation of the second stratum of the bifurcation set. It is the subset of parameters of the reduced versal deformation which correspond to functions with critical points in the boundary.

The formulas of versal deformations listed in Theorem 2.6 provide the explicit description of simple bifurcation diagrams and caustics.

Proposition. 1. The bifurcation diagram of $B_{2}$ in $c, \lambda$ plane is a smooth curve and a distinguished point in it. The bifurcation diagram of $B_{3} \subset \mathbf{R}^{3}$ is a cuspidal cylinder and a line in it which is tangent to the ridge. In general, the hypersurface component of the bifurcation diagram for $B_{k}$ series is a product of generalized swallow tail and a line. The second component is the maximal smooth submanifold passing through the vertex of the generalized swallow tail times a line.

2. The $B_{3}$ caustic is the union of two tangent lines, for $B_{4}$ this is a semicubic cylinder and a plane (the configuration is isomorphic to the discriminant of the standard $C_{3}$ boundary singularity).

3. The caustic of $F_{4}$ is the union of a Whitney umbrella which is the second component, and a smooth tangent surface which is the caustic of the $A_{2}$ singularity.

REMARK. The quasi-bifurcation diagrams cannot be free divisors in the ordinary sense because of the different dimensions of the components. However it is not hard to describe all vector fields tangent to the bifurcation diagram.

Proof of Theorem 2.6. By reducing the dimensions of $x$ by the stabilization lemma the proof splits into a few cases.

1. If $k=n-1$ the reduced function $\tilde{f}$ is a function in the variable $y$ only. The powers of the maximal ideal $C_{y} y^{m+1}$ form the splitting of the space $C_{y}$ of functions in $y$ into quasi-orbits. We get $B_{m}$ classes.

2. Assume that $k<n-1$. The rank of $d_{2} \tilde{f}$ can be 0,1 or 2 . In the latter case the second jet of $\widetilde{f}$ is equivalent to $y u$, where $u \in \mathbf{R}, v \in \mathbf{R}^{n-k-2}$ and $\widetilde{x}=(u, v)$. So, the function is quasi-equivalent to $h(y, u, v)=y u+g(y, \widetilde{x})$ with $g \in \mathcal{M}_{\widetilde{x}, y}^{3}$. 
Lemma 2.7. The germ $h(y, u, v)$ is quasi-equivalent to $y u+u p(v)+q(v)$ with $q(v) \in \mathcal{M}_{v}^{3}$, $p(v) \in \mathcal{M}_{v}^{2}$.

On the other hand, the same germ $h(y, u, v)$ is quasi-equivalent to $y^{2}-u^{2}+r(y, v)$ with $r(y, v) \in \mathcal{M}_{y, v}^{3}$.

An admissible family $h_{t}$ can be reduced to an admissible family of the form yu+ $u p_{t}(v)+q_{t}(v)$ and also of the form $y^{2}-u^{2}+r_{t}(y, v)$, by smoothly $t$-dependent family of quasi-equivalences. (We will call this reduction regular).

Proof. Since $\frac{\partial h}{\partial u}=y+\rho(y, \widetilde{x})$ with $\rho \in \mathcal{M}_{y, \widetilde{x}}^{2}$, the germ $h$ can be written as a sum of a function $h_{1}(y, \widetilde{x})$ which is affine in $y$, and a function $h_{2}(y, \widetilde{x})$ which belongs to the ideal $C_{y, \widetilde{x}}\left\{\frac{\partial h}{\partial u}\right\}^{2}$. Therefore, according to Lemma $2.3 h$ is right equivalent (via a change of $u$ coordinate only) to $h_{1}$. Apply now another right equivalence sending the coefficient in $h_{1}$ of $y$ to new $u$. Both are regular transformations. The function takes now the form $H(y, \widetilde{x})=y u+\varphi(u, v)$.

The square $H_{y}^{2}=u^{2}$ of the derivative $H_{y}=\frac{\partial H}{\partial y}$ belongs to the tangent space of the quasi-orbit of a function $H$. Hence for any $t \in[0,1]$ the monomial $u^{2}$ belongs to the tangent space of the deformation germ $H_{t}=y u+r(u, \widetilde{x}) u^{2} t+p(v) u+q(v)$, where $r, q, p$ are terms of the decomposition of $\varphi(u, v)$ in powers of $u$. Now Lemma 2.4 implies that $H$ is quasi-equivalent to the sum of $y u$ with the function affine in $u$, as required by the first claim. In the same way we see that $H$ is quasi-equivalent to a germ of the form $K=$ $y u+u^{2}+p(v) u+q(v)$. Setting now $x_{1}=u+\frac{1}{2}(y+p(v))$, we get $K=x_{1}^{2}-\frac{1}{4}(y+p(v))^{2}+q(v)$ as required by the second claim.

Lemmas 2.3, 2.4 imply the last claim. If germ $f$ depends on extra parameters then the reduction to the prenormal form can be chosen smooth in parameters.

3. If $k=n-2$ then $\widetilde{x} \in \mathbf{R}$ and the normal form of the function $h$ is simply $y u$. In particular, this is the case when $x, y \in \mathbf{R}^{2}$.

4. If $k=n-3$ and $\operatorname{rank}\left(d_{2} f\right)=n-2$ the second jet after stabilization is equivalent to $y^{2}$. So, the function is equivalent to $f=y^{2}+g(x, y), g \in \mathcal{M}_{x, y}^{3}$. Using Lemma 2.4 reduce the germ to the form $f=y^{2}+p(x) y+q(x)$. Add an extra stabilization variable $u$ and get $f_{*}=y^{2}-u^{2}+p(x) y+q(x)=-(y+u)^{2}+2(y+u) y+p(x) y+q(x)$. These operations are regular in the sense of Lemma 2.7. Put $\widetilde{u}=2(y+u)+p(x)$ and get $f_{*}=-\frac{1}{4} \widetilde{u}^{2}+\widetilde{u} y+\widetilde{u} p(x)-\frac{1}{4} p^{2}(x)+q(x)$, which is a stabilization of the prenormal form $\widetilde{f}=\widetilde{u} y+\widetilde{u} p(x)+\widetilde{q}(x)$ with $\widetilde{q}(x)=q(x)-\frac{1}{4} p^{2}(x)$. Lemmas 2.3, 2.4 imply that all these transformations are regular.

5 . Notice that, if $k \leq n-2$ and after the reduction $\operatorname{rank}\left(d_{2} \tilde{f}\right)=0$, the germ $\tilde{f}$ belongs to $\mathcal{M}^{3}(y, \widetilde{x})$ and fails to be simple. In fact, the 3 -jet of the quasi-equivalence orbit of a germ from $\mathcal{M}^{3}(y, \widetilde{x})$ coincides with the ordinary boundary equivalence orbits, since the difference is in $\left(\frac{\partial f}{\partial y}\right)^{2} \in M^{4}$. The action of diffeomorphisms on 3-jets of the germ $\tilde{f} \in \mathcal{M}^{3}$ coincides with the $\mathrm{Gl}(n-k, \mathbf{R})$ action by linear changes of variables. Even in the case of $n-k=2$ the four-dimensional space of degree 3 homogeneous polynomials in $y, \widetilde{x}$ split in a continuous family of at most 3-dimensional orbits of boundary preserving linear transformations. 
6. So, to get the complete classification of simple classes it remains to consider the quasi-equivalence within the space of germs in the form $\widetilde{f}=\widetilde{u} y+\widetilde{u} p(x)+\widetilde{q}(x), x \in \mathbf{R}^{m}$ with $p(x) \in \mathcal{M}_{x}^{2}$ and $q(x) \in \mathcal{M}_{x}^{3}$.

The germ $f$ is right equivalent (in the ordinary sense) to $\widetilde{u} Y+q(x)$, where $Y=y+p$, and hence is a stabilization of right class of $q$ only. Hence the right class of $q$ is a quasiinvariant of $f$. Simple $f$ can happen only for $m=1$ or $m=2$ when $q(x)$ is of the type $A_{k}, D_{k}, E_{k}$.

Now we may fix a simple germ $q(x)$ and consider an admissible deformation $f_{t}=$ $u y+u p_{t}(x)+q(x)$. The quasi-equivalence tangent space at fixed instant $t$ is the space of germs $u \frac{\partial p_{t}(x)}{\partial t}$ which admit a decomposition

$$
\begin{aligned}
u \frac{\partial p_{t}(x)}{\partial t}=\left(y+p_{t}(x)\right) A(u, x, y)+u y B(u, x, y) & +u^{2} K(u, x, y) \\
& +\sum_{i=1}^{m}\left(u \frac{\partial p_{t}(x)}{\partial x_{i}}+\frac{\partial q(x)}{\partial x_{i}}\right) S_{i}(u, x, y)
\end{aligned}
$$

with some smooth functions $A, B, K, S_{i}$.

Setting $y=-p_{t}(x)$ and keeping in the formula only homogeneous terms of degree 0 and 1 in $u$ we get

$$
u \frac{\partial p_{t}(x)}{\partial t}=u p_{t}(x) b(x)+\sum_{i=1}^{m}\left(u \frac{\partial p_{t}(x)}{\partial x_{i}}+\frac{\partial q(x)}{\partial x_{i}}\right) s_{i}(x)+\sum_{i=1}^{m}\left(\frac{\partial q(x)}{\partial x_{i}}\right) u r_{i}(x)
$$

for some smooth functions $b, s_{i}, r_{j}$. Equating terms of order 0 in $u$ we get

$$
\sum_{i=1}^{m}\left(\frac{\partial q(x)}{\partial x_{i}}\right) s_{i}(x)=0 .
$$

Due to acyclicity of Koszul complex for $q$ this implies that $s_{i} \in J_{f}$. Now the homogeneous terms linear in $u$ provide

$$
\frac{\partial p_{t}(x)}{\partial t}=p_{t}(x) b(x)+\sum_{i=1}^{m}\left(\frac{\partial q(x)}{\partial x_{i}}\right) \widetilde{r}_{i}(x) .
$$

Project the equality to a jet space of functions in $x$ of sufficiently high order, and then project to the local algebra $C_{x} / J_{q(x)}$. The tangent space becomes the principal ideal in $C_{x} / J_{q(x)}$ generated by $p(x)$. So an admissible family determines class of $p \in \mathcal{M}_{x}^{2}$ in the local algebra of $q$ up to a multiplication on an invertible factor. Hence simple singularity classes of $p$ are possible only for discrete ideal structure in $C_{x} / J_{q(x)}$. The only possibilities are $A_{k}$ classes with $q(x)=x_{1}^{k+1}$ and ideals generated by $x_{1}^{l}, 2 \leq l \leq k-1$, and $D_{k}$ classes for $q=x_{1} x_{2}^{2}+x_{1}^{k-1}$ with the ideal structure $\left\{x_{1}^{2}\right\} \supset\left\{x_{1}^{3}\right\} \ldots\left\{x_{1}^{k-2}\right\}$. The second order terms $x_{1}^{2}+a x_{1} x_{2}$ in the local algebra of $E_{6}$ (for $q=x_{1}^{4}+x_{2}^{3}$ ) generate a one-parameter family of principal ideals. This is the first case when the prenormal forms fail to be simple.

3. Lagrangian projections with boundary. Consider a Lagrangian submanifold $L$ of $T^{*} \mathbf{R}^{m}$ with a boundary $I$, which is an (isotropic) $(m-1)$-dimensional submanifold of $L$. 
A family of functions $f(x, y, \lambda)$ depending on parameters $\lambda$ defines a Lagrangian submanifold $L$ in $T^{*} Q, Q=\{\lambda\}$ by standard Hörmander formulas for generating families (provided that Morse non-degeneracy condition holds)

$$
L=\left\{(\lambda, \mu) \in \mathbf{R}^{m} \times \mathbf{R}^{m}: \exists(x, y) \in \mathbf{R}^{n}, \frac{\partial f}{\partial x_{i}}=\frac{\partial f}{\partial y}=0, \mu=\frac{\partial f}{\partial \lambda}\right\} .
$$

Critical points of $f$ which belong to $B=\{y=0\}$ correspond to the Lagrangian boundary $I$.

Standard arguments (see e.g. [1]) show that any germ of Lagrangian submanifold with boundary has a generating family. Notice that the families are defined not uniquely, at least up to stabilization and pseudo-equivalences. A discussion of this can be found in [6]. If a Lagrangian germ with boundary $L_{1}, I_{1}$ is mapped to another germ $L_{2}, I_{2}$ by a symplectomorphism of the ambient space, which preserve the Lagrangian fibration $\pi: T^{*} \mathbf{R}^{m} \rightarrow \mathbf{R}^{m}$ (the germs are called Lagrangian equivalent), then a generating family of $L_{1}, I_{1}$ is sent to a generating family of $L_{2}, I_{2}$ by a pseudo-equivalence.

The notions of stability and simplicity of Lagrangian submanifolds with boundary (with respect to Lagrange equivalence) are straightforward. The following statement is also a direct consequence of the classification of the quasi-simple classes and of the fact that for regular Lagrangian germs the radical of the ideal $\left\{\frac{\partial f}{\partial x}, \frac{\partial f}{\partial y}\right\}$ coincides with the ideal itself.

THEOREM 3.1. 1. A germ L, I is stable if and only if its arbitrary generating family is versal with respect to quasi-equivalence.

2. Any stable and simple projection of Lagrangian submanifold with a boundary is symplectically equivalent to the projection determined by one of the following generating families which are quasi-R+-versal deformations of the classes from Theorem 2.6

$$
\begin{array}{ll}
B_{2} \approx C_{2}: & x y+\lambda_{1} x, \\
B_{k}, k \geq 3: & x^{2}+y^{k}+\sum_{i=1}^{k-1} \lambda_{i} y^{i}, \\
F_{k, l}, 2 \leq k \leq l: & y x_{1}+x_{1}\left( \pm x_{2}^{k}+\sum_{i=1}^{k} \lambda_{i} x_{2}^{i-1}\right)+x_{2}^{l+1}+\sum_{j=k+1}^{l+k-1} \lambda_{j} x_{2}^{j-k}, \\
D_{k, l}, 2 \leq k \leq l-2: \quad & y x_{1}+x_{1}\left( \pm x_{2}^{k}+\sum_{i=1}^{k} \lambda_{i} x_{2}^{i-1}+\lambda_{k+1} x_{3}\right) \\
& +x_{2} x_{3}^{2} \pm x_{2}^{l-1}+\sum_{j=k+1}^{l+k-1} \lambda_{j} x_{2}^{j-k}+\lambda_{k+l} x_{3} .
\end{array}
$$

The theorem implies the description of stable simple caustics of Lagrangian submanifolds with boundary. In particular:

- The caustic of singularity $B_{k}$ is a union of a cylinder over generalized swallow tail (with one-dimensional generators) and a smooth hypersurface having smooth $(k-3)$ dimensional intersection with the first component. 
- The caustic of $F_{k, l}$ singularity is a union of a cylinder over a generalized swallow tail of type $A_{l}$ and an image of Morin stable mapping (generalized Whitney umbrella) being the set of common zeros of two polynomials of degrees $l$ and $k$.

- The caustic of $D_{k, l}$ singularity is a union of a cylinder over the caustic of $D_{k}$ singularity and a singular hypersurface which is an image of a deformation of a mapping from $\mathbf{R}^{2}$ to $\mathbf{R}^{3}$ with zero rank at the origin.

4. Quasi-projections. Another interesting example of non-standard equivalence relation is provided by a non-singular vector field $v$ or foliation in the space where a function or a complete intersection is defined.

Definition 4.1. Two functions $f_{i}: \mathbf{R}^{n} \rightarrow \mathbf{R}, i=1,2$, are called $v f$-equivalent if there is a diffeomorphism $\Theta: \mathbf{R}^{n} \rightarrow \mathbf{R}^{n}$ such that $f_{2}=f_{1} \circ \Theta$ and if $m$ is a critical point of $f_{1}$ then the linear part of $\Theta$ at $m$ maps the direction of the vector field $v$ to the direction of $v$ at the image $\Theta(m)$.

This equivalence takes an intermediate place between the standard right-equivalence and the right action of fibration preserving diffeomorphisms $\theta: \mathbf{R}^{n} \rightarrow \mathbf{R}^{n}, \theta_{*} v=h v$. In the simplest case of non-singular vector field $v=\frac{\partial}{\partial x}, x \in \mathbf{R}, \mathbf{R}^{n}=\{(x, y)\}, y \in \mathbf{R}^{n-1}$, which we assume to be the case throughout this section, diffeomorphisms $\theta$ preserve the fibration $\pi:(x, y) \mapsto y$, that is $\theta:(x, y) \mapsto(X(x, y), Y(y))$. The singularities of hypersurfaces with respect to this action were studied by Goryunov in [4]. It is related to the singularities of projections along fibers, and also to the theory of envelopes of moving wavefronts, that is to the space-time parameter depending families of functions, see [7]. The other application of this group is the classification of singularities of divergent diagrams of mappings and of parameter depending optimization problems [3]. The study of this intermediate group permits to understand better invariants of fiber preserving group action.

Similarly to the previous section, we introduce the infinitesimal version of the finer version of $v f$-relation, respecting regularity properties of deformation. It will be called quasi-projection equivalence. So, we use the following tangent space to the finer equivalence class of the function $f$

$$
T_{f}=\frac{\partial f}{\partial x} C_{x, y}+\frac{\partial f}{\partial y_{i}} \mathbf{K}
$$

where $\mathbf{K}$ is the module of vector functions $\mathbf{h}(x, y)$ the components $h_{i}$ of which satisfy the property $\frac{\partial h_{i}}{\partial x} \in J_{f}$, replacing by $\mathbf{K}$ the module of vector-functions $\left\{\mathbf{h}(x, y): \frac{\partial h}{\partial x} \in\right.$ $\left.\operatorname{Rad}\left(J_{f}\right)\right\}$ which enters the genuine $v f$-tangent space.

In fact, the condition that vector field

$$
u=a \frac{\partial}{\partial x}+b \frac{\partial}{\partial y}
$$

generated by a family of admissible diffeomorphisms preserves the direction of $v$ at some point means that the commutator $[v, u]$ is collinear to $v$ that is $\frac{\partial b}{\partial x}$ vanishes at that point.

Methods similar to that of Theorem 2.6 prove the following 
THEOREM 4.2. The simple quasi-projection classes of function germs are given by the following list:

$$
\begin{array}{ll}
A_{0}: & x \\
\widetilde{A}_{k}, \widetilde{D}_{k}, \widetilde{E}_{k}: & x^{2}+f(y), \\
C_{2}: & x y_{1} \pm y_{2}^{2} \pm \cdots \pm y_{n-1}^{2} \\
F_{n+1}: & x^{3}+ \pm y_{1}^{2} \pm \cdots \pm y_{n-1}^{2}
\end{array}
$$

$f(y)$ is a standard simple singularity class $A_{k}, D_{k}, E_{k}$.

REMARKs. 1. The fencing non-simple classes are $\widetilde{F}: x^{4}+a x^{2} y_{1}^{2}+ \pm y_{1}^{2} \pm \cdots \pm y_{n-1}^{2}$, and $C: x y_{1}+y_{2}^{3} \pm y_{3}^{2} \pm \cdots \pm y_{n-1}^{2}$.

2. The adjacency of the classes of low codimension in the plane $(x, y) \in \mathbf{R}^{2}$ is shown in the table:

$$
\begin{gathered}
A_{0} \leftarrow \widetilde{A}_{1} \leftarrow \widetilde{A}_{2} \leftarrow \widetilde{A}_{3} \leftarrow \quad \leftarrow \ldots \\
\uparrow \\
C_{2} \leftarrow F_{n+1} \leftarrow \widetilde{F}
\end{gathered}
$$

3. The contact quasi-projection classification coincides with the right one given in the theorem due to weighted homogeneity of the classes.

Acknowledgements. Partially supported by FBRF 080100157 and INTAS 0510000087805 grants.

\section{References}

[1] V. I. Arnold, Singularities of Caustics and Wave Fronts, Kluwer, Dordrecht 1990.

[2] J. Damon, A-equivalence and the equivalence of sections of images and discriminants, in: Singularity Theory and its Applications, Part I, Lecture Notes in Math. 1462, Springer, Berlin 1991, 93-121.

[3] A. A. Davydov, V. M. Zakalyukin, Classification of relative minima singularities, in: Geometry and Topology of Caustics - Caustics '98, Banach Center Publ. 50, Warszawa, 1999, $74-90$.

[4] V. V. Goryunov, Singularities of projections of complete intersections (Russian), in: Current Problems in Mathematics 22, Itogi Nauki i Tekhniki, Akad. Nauk SSSR, VINITI, Moscow, 1983, 167-206.

[5] S. Janeczko, On isotropic submanifolds and evolution of quasicaustics, Pacific J. Math. 158 (1993), 317-334.

[6] V. M. Zakalyukin, Singularities of isotropic submanifold projections, C. R. Acad. Sci. Paris Sér. I Math. 320 (1995), 1177-1182.

[7] V. M. Zakalyukin, Envelopes of families of wave fronts and control theory, Trudy Mat. Inst. Steklov. 209 (1995), 133-142; English transl.: Proc. Steklov Inst. Math. 209 (1995), $114-123$. 
